\title{
Amplitude Modulation of Frication Noise by Voicing Saturates
}

\author{
Jonathan Pincas and Philip J. B. Jackson
}

\author{
Centre for Vision, Speech \& Signal Processing, Univ. of Surrey, Guildford, Surrey GU2 7XH, UK. \\ [j.pincas, p.jackson] @surrey.ac.uk
}

\begin{abstract}
The two distinct sound sources comprising voiced frication, voicing and frication, interact. One effect is that the periodic source at the glottis modulates the amplitude of the frication source originating in the vocal tract above the constriction. Voicing strength and modulation depth for frication noise were measured for sustained English voiced fricatives using high-pass filtering, spectral analysis in the modulation (envelope) domain, and a variable pitch compensation procedure. Results show a positive relationship between strength of the glottal source and modulation depth at voicing strengths below $66 \mathrm{~dB}$ SPL, at which point the modulation index was approximately 0.5 and saturation occurred. The alveolar $[\mathrm{z}]$ was found to be more modulated than other fricatives.
\end{abstract}

\section{Introduction}

Fricatives are speech sounds produced by forcing air through a narrow constriction superior to the glottis, generating turbulence noise within the jet itself, or at/along a physical obstacle further downstream. English has voiceless and voiced fricatives at four places of articulation: labiodental, dental, alveolar and postalveolar, giving a total of eight fricative phonemes.

Voiced fricatives are generally distinguished by the presence of glottal and fricative sources, and this mixed excitation lends them their 'buzzy' quality. The characteristics of voiced frication do not arise simply from the linear combination of its component sources. The articulatory, aerodynamic and acoustic conditions required by and resulting from the simultaneous production of glottal vibration and frication noise raise the possibility of 'mutual interaction effects' [1]: the presence of each source causes the other to be changed in character from the case where it occurs in isolation. The focus of this paper, amplitude modulation (AM) of the frication component, is one such effect; others include mutual amplitude reduction [2], changes in fundamental frequency of voicing [3], and spectral changes in the voicing component (before, during and after frication) [4] and in the frication-noise component [5].

Although the presence of AM noise in voiced fricatives is widely acknowledged, the underlying mechanism is still not fully understood. During voiced frication, transglottal pressure and laryngeal tension conditions combine to maintain glottal vibration. The results of phonation are twofold and travel through the vocal tract at different speeds [6]: a jet of air leaving the glottis generates sound via pressure fluctuation, and sets up hydrodynamic motion (mean flow velocity). Amplitudemodulated frication is normally assumed to result from periodically pulsed flow through a fixed-area constriction [5]. A possible interpretation, then, is that the variations in airflow caused by the periodic interruptions of glottal vibration are responsible for modulation. Indeed, fluctuations in the amplitude of the noise source of up to $15 \mathrm{~dB}$ have been attributed to this mechanism [2]. The aerodynamic situation, however, is not so straightforward. Mechanical model studies have shown that airflow conditions along the vocal tract are complex, with the high degree of periodic airflow fluctuation immediately superior to the glottis being largely eroded further up the vocal tract [7]. Although it is thus hard to predict the patterns of airflow at any potential constriction in the vocal tract, it is unlikely that this mechanism is solely, or even partly, responsible for modulation. It is, in fact, more likely that AM is attributable to the interaction of the pressure wave created by phonation and the turbulent jet formation process at the fricative constriction [6]

Periodic, large-scale regularity in unstable flows is a common phenomenon in fluid mechanics. Although it has been shown that jets issuing from circular constrictions can exhibit large-scale regularity at increasing Reynolds numbers without any accompanying sound-pressure field [8], it appears that when a pressure wave at or near the natural Strouhal number of the jet is introduced, the cyclical fluctuation in the jet flow is significantly boosted. This is possible because unstable jet formation is sensitive to the presence of acoustic waves [8], which regularise, or force, turbulence generation.

For voiced fricatives, the forcing pressure wave is that set up by glottal vibration. This wave then interacts with the jet formation process at the supraglottal constriction, producing a periodically-fluctuating jet. In the majority of cases, the creation of amplitude-modulated noise probably depends heavily on the jet striking a downstream obstacle, such as the lips or teeth. Phase differences between the glottal and noisemodulation signals reported by Jackson and Shadle support this interpretation [6].

There has been little quantitative study devoted to the acoustic characteristics of AM noise in fricatives. For fricatives embedded in fluent speech nonsense words, Pincas and Jackson found that modulation depth tracked voicing strength quite closely and that the voiced fricative $[\mathrm{z}]$ was generally more heavily modulated than others [1]. Jackson and Shadle also published limited data relating to amplitude of modulation in various voiced fricatives [6]: their results range from $0 \mathrm{~dB}$ in the case of $[\beta]$ to $2 \mathrm{~dB}$ in the case of $[\mathrm{z}]$; modulation for the other fricatives tended to cluster around $1 \mathrm{~dB}$.

This study aims to extend our current knowledge of the AM noise generation process by exploring the relationship between the forcing glottal wave and modulation depth. The data obtained is also apt for integration into a speech synthesis system.

\section{Method}

\subsection{Speech Data Acquisition}

The Corpus: Sustained English voiced fricatives ([v,ð,z,z]) were produced by 16 speakers $(12 \mathrm{M}, 4 \mathrm{~F})$. Each fricative was produced separately with voicing at 125, 150 and $175 \mathrm{~Hz}$. Each fricative-pitch combination was preceded by a calibration tone 
played through a loudspeaker and a short (2-s) pause allowing the subject to attain the correct voicing pitch. Two repetitions of each combination were performed. The first was an uninterrupted fricative where the subject smoothly adjusted loudness from the quietest fricative they could produce to relatively loud, and back again ( $\sim 3 \mathrm{~s}$ in total). The second repetition consisted of three separate sustained fricative bursts with gradually increasing amplitude, each lasting approximately $1 \mathrm{~s}$. For each speaker 24 recordings were made (4 frics $\times 3$ pitches $\times 2$ reps). Recording: Speech audio and electroglottograph (EGG) signals were captured simultaneously on PC by a Creative Labs Audigy soundcard via a Sony SRP-V110 desk (2 channels at $44.1 \mathrm{kHz}$ with 16-bit resolution): mono audio from a Beyerdynamic M59 microphone, and EGG from a Laryngograph Lx Proc PCLX with adult-sized electrodes. The microphone was calibrated by comparing a $1 \mathrm{kHz}$ tone played through a loudspeaker at $10 \mathrm{~cm}$ to an SPL measurement made with a Brüel and Kjær Type 2240 SPL meter at the same distance. Subjects placed their head in a support to minimise movement throughout recording and the calibrated microphone was placed $10 \mathrm{~cm}$ away, at lip level and at approximately $45^{\circ}$ to the subject's line of sight. The EGG signal provided accurate pitch information which was used by the modulation depth estimation algorithm.

\subsection{Measuring Modulation Depth}

Fundamentals of AM: Modulation depth, $m$, is most often given in standard index form, which can be conceptualised as the fraction of the carrier signal that the modulated signal varies by, e.g., if $m=0.5$, then the signal fluctuates by $50 \%$ above and below its original, unmodulated value. In most applications of AM (such as in acoustics or telecommunications), $m$ ranges from 0 (unmodulated) to 1 (completely modulated).

In AM, the amplitude of the carrier signal, $w(n)$, is modified by a modulating signal, $a(n)$, to produce an amplitudemodulated signal, $x(n)=w(n) a(n)$. In the case of a periodic modulating signal, $a(n)$ takes the form of a fundamental sinusoid of frequency $f_{0}$ plus its harmonics. Thus, we have

$$
x(n)=w(n)\left[1+\sum_{h=1}^{H} m_{h} \cos \left(\frac{2 \pi h f_{0} n}{f_{S}}+\phi_{h}\right)\right],
$$

where $h \in 1 . . H$ are the harmonics, $m_{h}$ is the modulation index at $h f_{0}, f_{s}$ is the sampling frequency and $\phi_{h}$ is an arbitrary phase shift which we assume to be constant. With purely sinusoidal amplitude modulation $(H=1)$, the signal $a(n)$ is completely specified by the $f_{0}$ component, i.e., by $m_{1}$ and $\phi_{1}$. In natural voiced fricatives, the underlying modulation shape is unlikely to be purely sinusoidal. Here, however, we will mainly be concerned with modulation at $f_{0}$, and so for ease of reference we will refer to $m_{1}$ as $m_{f 0}$. Where we refer to higher modulation harmonics, they are designated $m_{2 f 0}, m_{3 f 0}$ etc.

Estimating $m_{f 0}$ : In the case of modulated broadband noise, the carrier signal $w(n)$ takes the form of a random variable which we model as Gaussian white noise and the signal $x(n)$ is fully specified by Equation 1. To estimate $m_{f 0}$ we first take the instantaneous magnitude of the signal: $|x(n)|=|w(n)| a(n)$, which contains a periodic component at $f_{0}$, the strength of which is directly proportional to $m_{f 0}$. Hence we compute its Fourier transform, $\bar{X}(k)=\mathcal{F}\{|x(n)|\}$, first applying a Hamming window and zero-padding to $N$ points $\left(2^{15}\right)$ :

$$
\bar{X}(k)=\mathcal{F}\{|w(n)|\} \otimes\left[\delta(0)+\sum_{h=1}^{H} \frac{m_{h}}{2}\left(\delta\left( \pm h k_{0}\right) \mathrm{e}^{ \pm j \phi_{h}}\right)\right],
$$

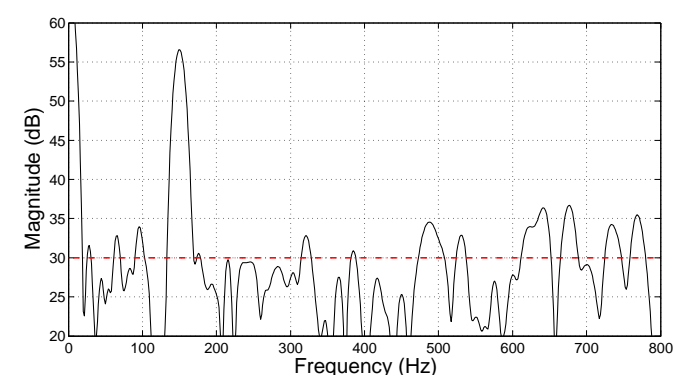

Figure 1: Modulation spectrum for $100 \mathrm{~ms}$ of broadband noise $\left(f_{0}=150 \mathrm{~Hz}, m_{f 0}=0.5\right)$. Dashed line indicates noise floor.

where $\otimes$ denotes convolution, $\delta(\cdot)$ the Dirac delta function, and $k_{0}=N f_{0} / f_{S}$ is the frequency bin that contains $f_{0}$. Figure 1 presents a synthetic example of the modulation spectrum, $\bar{X}(k)$, for white noise modulated at $f_{0}=150 \mathrm{~Hz}$, where the spike occurs. ${ }^{1}$ Finally, $m_{f 0}$ can be calculated by comparing the magnitudes of the Fourier coefficients at d.c. and $f_{0}$ :

$$
m_{f 0}=2 \frac{\left|\bar{X}\left(k_{0}\right)\right|}{|\bar{X}(0)|},
$$

where the factor of two leads to an estimate of the standard modulation index. Clearly, where $m_{2 f 0}, m_{3 f 0}$ etc. are required in place of $m_{f 0}, k_{0}$ is replaced by the relevant integer multiple.

Isolating the frication noise: In the case of voiced fricatives, the carrier noise $w(n)$ would not be white, but coloured (filtered) depending on the fricative place of articulation. A further complicating factor is the presence of low-frequency voicing and excited formants mixed with the frication noise. Given that periodically-excited formants are damped oscillations pulsed at $f_{0}$, the presence of periodic energy normally serves to attenuate aperiodic modulation depth, unless the pulses are perfectly in phase with the bursts of frication noise. Since we are interested only in modulation of the frication noise, it is paramount that we successfully isolate the aperiodic component before applying the procedure outlined above. Efficient removal of periodic components is achieved by high-pass (HP) filtering with a cutoff frequency, $f_{\mathrm{HP}}$. However, since HP filtering also removes noise components below $f_{\mathrm{HP}}$, we would effectively only be measuring modulation for frication noise above $f_{\mathrm{HP}}$. Inspection of spectrograms suggests that modulation is unlikely to be uniform across the spectrum: noise in high-frequency regions looks to be more modulated than in lower regions, where it is more concentrated. Thus, biasing measurement to noise in the upper frequency bands will lead to an overestimation of modulation depth with regard to the full spectrum of frication noise, which is our ultimate object of interest. To balance the need for effective removal of periodic components and accurate estimation of modulation depth, we experimented with a 40th-order HP filter at six cutoff frequencies, $f_{\mathrm{HP}} \in\{0.7,1.4,2.7,4.5,8.4$ and $11.5 \mathrm{kHz}$.

Variable pitch: Although the processing window employed was short enough to exclude major changes in fundamental frequency, pitch variation within a window would lead to modulation energy being spread around $f_{0}$. To compensate for variable pitch, we based our estimate $\hat{m}_{f 0}$ on the area of the spike at $k_{0}$ (see Fig. 1). Upper and lower extremes of the base of the spike, $k_{L}$ and $k_{U}$, and hence its width, are dictated by the noise floor,

\footnotetext{
${ }^{1}$ Modulation does not alter the flatness of a white noise spectrum.
} 


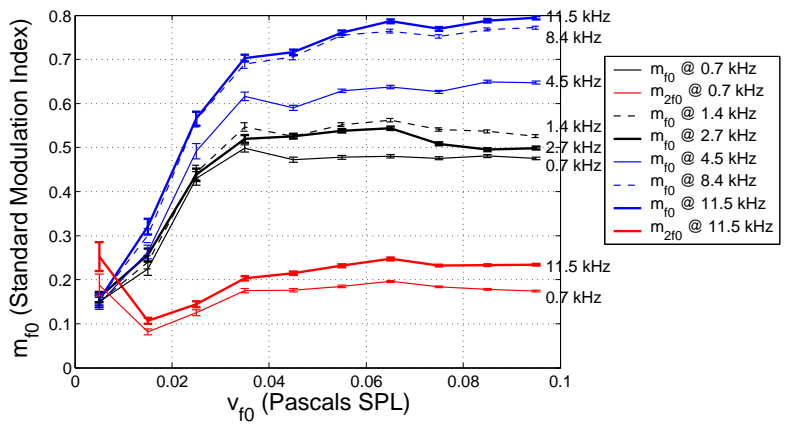

Figure 2: Modulation depths at the fundamental frequency $\hat{m}_{f 0}$ (blue/black lines) and second harmonic $\hat{m}_{2 f 0}$ (red lines) versus voicing strength $v_{f 0}$ for various high-pass filter cutoff frequencies, $f_{\mathrm{HP}}$. Data are means of values falling within $\pm 0.005 \mathrm{~Pa}$ bins from all tokens. Error bars show 95\% confidence intervals.
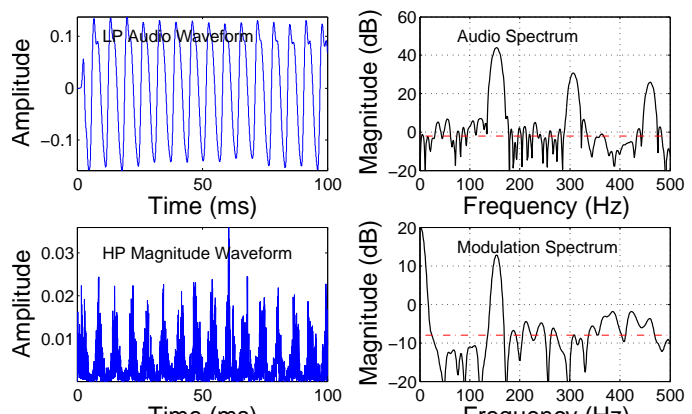

Time (ms) Frequency $(\mathrm{Hz}$

Figure 3: Illustration of the harmonic structure of $d(n)$ and $a(n)$ for $100 \mathrm{~ms}$ of the fricative $[\mathrm{z}]$ spoken by JS $\left(f_{0} \approx 150 \mathrm{~Hz}\right)$. Top left: audio waveform low-pass filtered at $1 \mathrm{kHz}$. Top right: audio spectrum up to $500 \mathrm{~Hz}$. Bottom left: magnitude waveform high-pass filtered at $9 \mathrm{kHz}$. Bottom right: its modulation spectrum. Dashed lines in spectra indicate noise floor.

$\theta \propto \sigma / \sqrt{N}$ where $\sigma^{2}$ is the variance of the noise and $N$ the number of samples in the analysis window. Thus, a measure equivalent to that of Eq. 2 based on the spike area is calculated:

$$
\hat{m}_{f 0}=\left(\frac{\sum_{k=k_{L}}^{k_{U}}|\bar{X}(k)|^{2}-\hat{\theta}^{2}}{K\left(|\bar{X}(0)|^{2}-\hat{\theta}^{2}\right)}\right)^{1 / 2}
$$

where $\hat{\theta}$ is the estimated level of the noise floor, and $K=k_{U}-$ $k_{L}+1$ is the number of points that fall under the spike.

Processing Conditions: Choice of window size is a trade-off between optimising modulation depth resolution and minimising effects of pitch glides. Simulations using synthesised signals proved $100 \mathrm{~ms}$ to be suitable. So, $\hat{m}_{f 0}$ was estimated with a $100 \mathrm{~ms}$ window, and a $5 \mathrm{~ms}$ step size. The required values of $f_{0}$ were obtained from spectral analysis of the EGG signal using the same parameters. For later comparison, voicing strength $v_{f 0}$ was defined as the amplitude of the spectral component at $f_{0}$ in the audio signal prior to filtering.

\section{Results and Discussion}

The $\hat{m}_{f 0} / v_{f 0}$ relationship: In Fig. 2, readings for all speakers, fricatives, pitch levels and repetitions are combined. Results are presented for all HP cutoff frequencies. To explore the re- lationship between modulation depth and voicing strength, the $v_{f 0}$ range $0.01-0.1 \mathrm{~Pa}$ SPL (up to $74 \mathrm{~dB}$ SPL) was split into 10 equally-spaced bins and readings within each bin averaged.

We begin by considering $\hat{m}_{f 0}$, the modulation depth at the fundamental, depicted by the black and blue lines in Fig. 2. Although data for very low voicing strengths was sparser, all bins have $95 \%$ confidence intervals narrower than 0.05 (modulation index), which is similar to predicted estimation error. The relationship between $\hat{m}_{f 0}$ and $v_{f 0}$ is non-linear for all values of $f_{\mathrm{HP}}$, with saturation occurring at approximately $v_{f 0}=0.04 \mathrm{~Pa}$ (at $10 \mathrm{~cm}$ ) for all but the $f_{\mathrm{HP}}=11.5 \mathrm{kHz}$ case. At the point of saturation, modulation index varies between approximately 0.5 (for $f_{\mathrm{HP}}=700 \mathrm{~Hz}$ ) and 0.7 (for $f_{\mathrm{HP}}=11.5 \mathrm{~Hz}$ ). At lower voicing strengths the curve rises almost linearly, with an increase in modulation index of between 0.12 (for $f_{\mathrm{HP}}=700 \mathrm{~Hz}$ ) and 0.18 (for $f_{\mathrm{HP}}=11.5 \mathrm{~Hz}$ ) for every $0.01 \mathrm{~Pa}$ increase in $v_{f 0}$.

Effect for HP-cutoff frequency: The curves for different values of $f_{\mathrm{HP}}$ appear to support the initial observations mentioned in Sec. 2.2. At a HP filter cutoff of $700 \mathrm{~Hz}$, we expect a certain amount of voicing energy mixed with frication noise and thus probable underestimation of true modulation; Fig. 2 (thin black line) bears this out. Raising the cutoff to $1.4 \mathrm{kHz}$ (dashed black line) eliminates most periodic energy without excluding a significant amount of frication noise and this modulation depth is better estimated. Notice how raising the cutoff further to $2.7 \mathrm{kHz}$ (thick black line) produces little difference: periodic energy is already mostly eliminated and the bulk of the frication energy, for most places of articulation, remains above the cutoff frequency. However, raising $f_{\mathrm{HP}}$ further to $4.5 \mathrm{kHz}$ (thin blue line) and $8.4 \mathrm{kHz}$ (dashed blue line) produces overestimation as measurement is biased to the more deeply modulated noise in the higher-frequency region. Raising the cutoff from 8.4 to $11.5 \mathrm{kHz}$ (thick blue line) has little effect as most of the concentrated noise has already been excluded. We conclude, then, that HP filtering at approximately $1.5-3 \mathrm{kHz}$ is suitable as pre-processing to the estimation procedure described in Sec. 2.2 and hence these results best reflect real modulation depths for voiced fricatives.

Harmonic structure of $a(n)$ : The aeroacoustic processes that produce AM noise in voiced fricatives might be thought of as follows: a forcing glottal wave, $d(n)$, interacts with a noise generation process to produce AM noise near the fricative constriction. Following reflections within the vocal tract, the noise radiates as the voiced fricative signal, $x(n)$. The shape of $x(n)$ 's envelope is described by the underlying modulating signal $a(n)$, which has a component $m_{f 0}$ at the fundamental. In relating $d(n)$ to $a(n)$, note that the results discount the hypothesis that $d(n)$ is equal to $a(n)$ (i.e., that the underlying modulation is identical in shape to the forcing wave that initiated it). This is manifested by the saturation in the relationship between the fundamental components of $d(n)$ and $a(n): v_{f 0}$ and $m_{f 0}$ respectively. Yet, the full $d(n)$ to $a(n)$ mapping requires further clarification.

Our observations confirm that even the most strongly modulated frication noise shows no detectable components above the second harmonic (i.e., only a fundamental and second harmonic are present) and in many cases the harmonic is so weak as to blend into the background fluctuations, leaving a fundamental only. This is true even when the forcing wave shows significant harmonic structure. Figure 3 gives an example of such a situation for a token of $[\mathrm{z}]$ taken from the corpus. Notice how the harmonic structure of $d(n)$ (top right) is not preserved in the modulation of the noise (bottom right). 


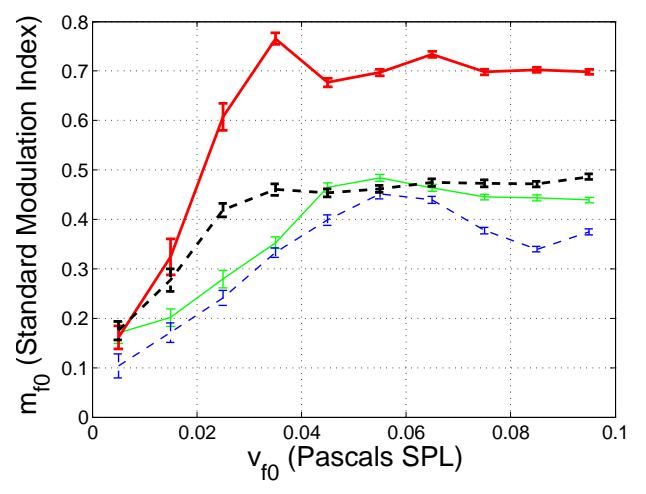

Figure 4: Modulation depth $\hat{m}_{f 0}$ as a function of voicing strength $v_{f 0}$ for fricatives [v] (thin blue dashed ); [ð] (thin green solid); [z] (thick red solid) and [3] (thick black dashed). Data are means across speakers, pitches and repetitions $\left(f_{\mathrm{HP}}=2.7 \mathrm{kHz}\right)$. Binning and error bars as per Fig. 2.

\begin{tabular}{cc|cc|cc|cc}
\hline \hline Sbj. & $\hat{m}_{f 0}$ & Sbj. & $\hat{m}_{f 0}$ & Sbj. & $\hat{m}_{f 0}$ & Sbj. & $\hat{m}_{f 0}$ \\
\hline JP & 0.44 & MZ & 0.73 & PJ & 0.73 & AT $^{*}$ & 0.56 \\
AG & 0.49 & SA & 0.60 & MD & 0.39 & FB $^{*}$ & 0.62 \\
GC & 0.63 & GM & 0.61 & AL & 0.87 & RG $^{*}$ & 0.65 \\
JS & 0.63 & RK & 0.60 & LM & 0.58 & LE $^{*}$ & 0.60 \\
\hline \hline
\end{tabular}

Table 1: Subjects' mean $\hat{m}_{f 0}$ at $v_{f 0}=0.05-0.06 \mathrm{~Pa} .{ }^{*}$ female.

Referring back to Figure 2 (red lines), it is clear that as $v_{f 0}$ increases, a significant modulation harmonic $\hat{m}_{2 f 0}$ does arise (for clarity we show harmonics for $f_{\mathrm{HP}}=700 \mathrm{~Hz}$ and $f_{\mathrm{HP}}=11.5 \mathrm{kHz}$ only as high-pass cutoff frequency appears to make little difference). Although our results cannot rule out the possibility that this harmonic is caused by harmonics in the forcing wave, it seems more likely that the results are analogous to the similarly-shaped curves obtained by Crow and Champagne in a comparable study using turbulent jets forced by a pure sinusoid from a loudspeaker [8].

Perceptual considerations: Along with the fact that the modulation depth of any higher harmonic is likely to be relatively small, it is also likely to be less perceptible to listeners. It is unclear exactly how modulated noise in voiced fricatives will be perceived due to complicating factors such as their short length and presence of the low-frequency voicing signal (psychoacoustic studies have generally used $>500$-ms stimuli with noise only, making them of limited relevance). However, it is known that modulation of noise and tones is increasingly harder to detect at higher frequencies. For broadband noise modulated in the $f_{0}$ range typical of speech, sensitivity to modulation decreases at approximately $3 \mathrm{~dB}$ per octave [9]. Thus, for a modulating $f_{0}=125 \mathrm{~Hz}$, the detection threshold at the second harmonic would be $m_{2 f 0} \approx 0.18$, but lower at the fundamental, $m_{f 0} \approx 0.13$.

Effect for place of articulation: Differences amongst the four English fricatives are illustrated in Figure 4, which uses a similar binning procedure as in Fig. 2. With fewer readings in each bin, confidence intervals are generally wider. For all four fricatives, the relationship is of the type illustrated in Fig. 2, although its parameters differ for each place of articulation. The curve for [z] (thick red solid line) stands out: it is the quickest to saturate (at $v_{f 0} \approx 0.035$ ) and does so at a higher modulation depth. Fur- thermore, the transition from the rising, linear part of the curve to the saturated part is more abrupt than other fricatives. The high modulation depth at saturation for [z] in Fig. 4 is common to most speakers: 14 of our 16 subjects have [z] as the most heavily modulated fricative at $v_{f 0}=0.05 \mathrm{~Pa}^{2}$ These findings echo our previous results for $[\mathrm{z}]$ in fluent speech [1].

Effect for speaker: The $v_{f 0}-\hat{m}_{f 0}$ curves saturate at similar levels for all subjects, $v_{f 0} \approx 0.04 \mathrm{~Pa}$ ). Table 1 compares mean $\hat{m}_{f 0}$ (4 frics, 2 reps) at $0.05 \leq v_{f 0}<0.06 \mathrm{~Pa}$ (to allow for speakers with later saturation). The values vary significantly at saturation across subjects (overall mean 0.71). Individual differences in degree of modulation may be attributed to varying articulatory configurations across speakers. As such, modulation could correspond to some aspect of voice quality.

\section{Conclusion}

In voiced fricatives, phonation provokes AM of frication noise. A technique was developed to estimate the depth of modulation and applied to HF noise from sustained fricatives. Modulation depth rose approximately linearly with voicing strength for low voicing levels $(<66 \mathrm{~dB}$ SPL); it saturated at a similar voicing level for different fricatives and speakers, although its value at this point varied. In particular, [z] was most deeply modulated. Previous perceptual studies of modulated noise suggest that our observed levels of modulation are detectable. Further work could establish how amplitude-modulated noise in fricatives serves as phonetic cue or voice-quality characteristic.

\section{References}

[1] J. Pincas and P.J.B. Jackson, "Acoustic correlates of voicing-frication interaction in fricatives," in Proc. 'From Sound to Sense', MIT, Cambridge MA, USA, June 2004.

[2] K.N. Stevens, "Airflow and turbulence noise for fricatives and stop consonants: Static considerations," J. Acoust. Soc. Am., vol. 50, no. 4, pp. 1180-1192, 1971.

[3] A. Löfqvist, T. Baer, N.S. McGarr and R.S. Story, "The cricothyroid muscle in voicing control," J. Acoust. Soc. Am., vol. 85, no. 3, pp. 1314-1321, 1989.

[4] A. Löfqvist, L.L. Koenig, and R.S. McGowan, "Vocal tract aerodynamics in $/ \mathrm{aCa} /$ utterances: Measurements," Speech Communication, vol. 16, pp. 50-66, 1995.

[5] C.H. Shadle, "Modelling the noise source in voiced fricatives," in Proc. 15th Int. Cong. on Acoustics, vol. 3, 1995.

[6] P.J.B. Jackson and C.H. Shadle, "Frication noise modulated by voicing, as revealed by pitch-scaled decomposition," $J$. Acoust. Soc. Am., vol. 108, no. 4, pp. 1421-1434, Oct. 2000.

[7] A. Barney, C.H. Shadle, and P. Davies, "Fluid flow in a dynamic mechanical model of the vocal folds and tract. 1 . measurements and theory," J. Acoust. Soc. Am., vol. 105, no. 1 , pp. 444-455, 1999.

[8] S. Crow and F. Champagne, "Orderly structure in jet turbulence," J. Fluid Mech., vol. 48, no. 3, pp. 547-591, 1971.

[9] N. Viemeister, "Temporal modulation transfer functions based upon modulation thresholds," J. Acoust. Soc. Am., vol. 66, no. 5, pp. 1364-1380, 1979.

\footnotetext{
${ }^{2}$ In contrast, saturation points and levels for the remaining fricatives, whilst relatively similar and consistently distinct from $[\mathrm{z}]$, vary for each speaker with no clear pattern. This could be explained by articulatory configurations varying less across speakers for $[\mathrm{z}]$, but more for the other fricatives which tend either to cause difficulty (e.g., [3], quite rare in English) or to be produced in a variety of ways (e.g., [ð], varies in degree of tongue protrusion). The slightly narrower confidence intervals for $[\mathrm{z}]$ at higher voicing strengths concur.
} 